sensitivity, specificity, positive predictive value, and negative predictive value of vessel wall imaging in identifying unstable aneurysms were $87.2 \% \quad(81.8-91.5), \quad 61.1 \% \quad(56.8-65.3)$, $46.5 \% \quad(43.5-49.4)$ and $92.5 \%$ (89.6-94.7), respectively. A statistically significant association between vessel wall enhancement and aneurysm instability is once again demonstrated. The lack of wall enhancement is a strong predictor of aneurysm stability. Vessel wall imaging may represent a useful tool in guiding the management of intracranial aneurysms.

Disclosures A. Larson: None. G. Lanzino: None. W. Brinjikji: None.

\section{E-190 DETERMINING THE OPTIMAL TIMING OF DUAL ANTIPLATELET THERAPY FOR FLOW DIVERSION}

${ }^{1} V$ Ban*, ${ }^{2} \mathrm{M}$ Pernik, ${ }^{1} \mathrm{~T}$ Binyamin, ${ }^{1} \mathrm{~J}$ Corona, ${ }^{1} \mathrm{Y}$ Kim, ${ }^{1} \mathrm{R}$ de Oliveira Sillero, ${ }^{3} \mathrm{R}$ Novakovic, ${ }^{3} \mathrm{G}$ Pride, ${ }^{3} \mathrm{~J}$ Barr, ${ }^{1} \mathrm{~J}$ White, ${ }^{1} \mathrm{H}$ Batjer, ${ }^{1} \mathrm{~B}$ Welch. ${ }^{1}$ Neurological Surgery, UT Southwestern, Dallas, TX; ${ }^{2}$ UT Southwestern, Dallas, TX; ${ }^{3}$ Radiology, UT Southwestern, Dallas, TX

\subsection{6/neurintsurg-2020-SNIS.221}

Introduction The flow diverter is a unique and important tool in the endovascular treatment of aneurysms. However, its use requires patients to be on dual antiplatelet therapy to prevent thromboembolic complications. The duration of dual antiplatelet therapy has not been standardized due to the relative risks of thromboembolic (too short) and hemorrhagic (too long) complications. The objective of this study is to compare the thromboembolic and hemorrhagic complication rates of patients on a shorter versus a longer course of dual antiplatelet therapy.

Methods Patients undergoing flow diversion were prospectively enrolled in an institutional registry. After 3 to 6 months of dual antiplatelet therapy, patients were converted to aspirin $325 \mathrm{mg}$ up to the 12 month period. Patients on dual antiplatelet therapy for $<100$ days were included in the short cohort while those on dual antiplatelet therapy for $\geq 100$ days were included in the long cohort. The proportions of thromboembolic and hemorrhagic complications in these respective cohorts were compared using the Fisher's exact test.

Results A total of 110 cases were eligible (mean age: 56.7 years). The majority were female $(81.8 \%)$ and received the Pipeline Embolization Device (83.6\%). 7.3\% of patients presented with ruptured aneurysms. More than 1 flow diverter was required in $7.3 \%$ of cases. The majority $(90.9 \%)$ of the dual antiplatelet regimen involved aspirin $325 \mathrm{mg}$ and clopidogrel $75 \mathrm{mg}$. Most patients were on dual antiplatelet therapy between 3-6 months in duration prior to transitioning to aspirin monotherapy $(325 \mathrm{mg})$. In the shorter duration cohort, the thromboembolic complication rate was $9.3 \%$ compared to $12.5 \%$ in the longer duration cohort $(p=0.76)$. Similarly, the hemorrhagic complication rate was $5.6 \%$ in the short duration cohort compared to $14.3 \%$ in the longer duration cohort $(\mathrm{p}=0.20)$.

Conclusion A shorter duration of dual antiplatelet therapy after flow diversion was not associated with a higher thromboembolic complication rate. While the duration of antiplatelet therapy should be personalized for each patient, transitioning to monotherapy after 3 months is likely safe.

Disclosures V. Ban: None. M. Pernik: None. T. Binyamin: None. J. Corona: None. Y. Kim: None. R. de Oliveira Sillero: None. R. Novakovic: None. G. Pride: None. J. Barr: None. J. White: None. H. Batjer: None. B. Welch: 2; C; Medtronic, Stryker, MicroVention. 6; C; Peter Lazic.

\section{E-191 THE SAFETY AND EFFECTIVENESS OF THE LVIS STENT FOR THE TREATMENT OF ACUTELY RUPTURED INTRACRANIAL ANEURYSMS WITHIN 24 HOURS: A MULTICENTER RETROSPECTIVE STUDY}

${ }^{1} \mathrm{~B}$ Zhao*, ${ }^{2} \mathrm{X}$ Wang, ${ }^{3} Y$ Chen, ${ }^{4} \mathrm{~J} C a i,{ }^{5} \mathrm{D}$ Jin, ${ }^{1} \mathrm{~J}$ Wan, ${ }^{1} Y$ Pan, ${ }^{6} \mathrm{G}$ Mao. ${ }^{1}$ Neurosurgery, Renji Hospital, Shanghai Jiaotong University School of Medicine, Shanghai, China; ${ }^{2}$ Neurosurgery, The Second Hospital of NanChang University, Nanchang, China; ${ }^{3}$ Neuroradiology, The first Hospital of Wenzhou Medical University, Wenzhou, China; ${ }^{4}$ Neurosurgery, Linyi People's Hospital, Linyi, China; ${ }^{5}$ Neurosurgery, Dalian Central Hospital, Dalian, China; ${ }^{6}$ Neurosurgery, The Second Hospital of Nanchang University, Nanchang, China

\subsection{6/neurintsurg-2020-SNIS.222}

Background and Purpose Stent-assisted coiling is a feasible and effective treatment of ruptured aneurysms and is increasingly used in acutely ruptured aneurysms. The Low Profile Visualized Intraluminal Support (LVIS) stent is a self-expanding, retrievable, single-wire braid microstent system. Very small numbers of acutely ruptured aneurysms are reported. We aimed to evaluate the safety and effectiveness of the LVIS stent for the treatment of ruptured intracranial aneurysms within 24 hours of ictus compared with treatment between 25 and 72 hours.

Methods This was a multicenter retrospective study of acutely ruptured intracranial aneurysms treated with the LVIS stents. Acutely ruptured aneurysms were treated with LVIS stentassisted coiling within 72 hours after the initial subarachnoid hemorrhage. 110 consecutive patients with ruptured aneurysms underwent LVIS stent-assisted coiling from January 2017 to December 2017. The timing of treatment was grouped into the treatment within 24 hours of ictus and the treatment between 25 and 72 hours. Baseline characteristics, perioperative complications, angiographic results, and clinical outcomes were compared between the two groups.

Results A total of 101 patients with 101 acutely ruptured aneurysms were included in this study. $16(15.8 \%)$ patients had multiple aneurysms. $49(48.5 \%)$ patients underwent stentassisted coiling within 24 hours. There were no statistically significant differences in age, sex, WFNS grade, Fisher grade, aneurysm location, and aneurysm characteristics. The intraoperative complications occurred in 7 patients $(6.9 \%)$ and postoperative ischemia occurred in 5 patients (5\%). Perioperative complications occurred in $2(4.1 \%)$ patients treated within 24 hours compared with those in $10(19.2 \%)$ treated between 25-72 hours $(\mathrm{P}=0.019)$. The multivariate logistic regression analysis of predictor of perioperative complications showed that preoperative WFNS grade $(\mathrm{P}=0.017)$ and timing of treatment $(\mathrm{P}=0.042)$ were independent predictors of perioperative complications after stenting. The total aneurysm occlusion rate 